\title{
ON THE SYNCHRONY OF EMPIRICAL MODE DECOMPOSITIONS WITH APPLICATION TO ELECTROENCEPHALOGRAPHY
}

Justin Dauwels

\author{
Amari Research Unit \\ Brain Science Institute RIKEN \\ Wako-shi, Saitama, Japan \\ justin@dauwels.com
}

\author{
Tomasz M. Rutkowski, François Vialatte, Andrzej Cichocki
}

\begin{abstract}
A novel approach to measure the interdependence of time series is proposed, based on the alignment ("matching") of their Huang-Hilbert spectra. The method consists of three steps: first, empirical modes are extracted from the signals; those functions carry non-linear and non-stationary components in frequency limited bands. Second, the empirical modes are Hilbert transformed, resulting in very sharply localized ridges in the timefrequency plane; the obtained time-frequency representations are known as Huang-Hilbert spectra. At last, the latter are pairwise aligned by means of the stochastic-event synchrony method (SES), a recently proposed procedure to match pairs of multi-dimensional point processes. The level of similarity of two Huang-Hilbert spectra is quantified by three parameters: timing and frequency jitter of coincident ridges, and fraction of non-coincident ridges.

The proposed method is used to detect steady-state visually evoked potentials (SSVEP) in electroencephalography (EEG) signals; numerical results indicate that the method is vastly more sensitive to SSVEP than classical synchrony measures, and therefore, it may prove to be useful in applications such as brain-computer interfaces. Although the paper mostly deals with EEG, the presented synchrony measure may also be applied to other kinds of time series.
\end{abstract}

Index Terms - Electroencephalography, Time-frequency analysis, Synchronization, Spectral analysis, Hilbert transforms

\section{INTRODUCTION}

Quantifying the interdependence between time series is an important yet challenging problem. Although it is straightforward to quantify linear dependencies, the extension to non-linear correlations is far from trivial. A variety of approaches have been proposed, stemming from research fields as diverse as physics, statistics, signal processing, and information theory (see, e.g., [1] and [2]).

In this paper, we propose a novel approach to quantify synchrony between signals; it measures the similarity of the so called Huang-Hilbert spectra, which represent the instantaneous amplitude and frequency of empirical modes extracted from the original signals. In more classical time-frequency transformations such as (discrete and continuous) wavelet transforms, the set of time-frequency basis functions ("wavelets") is fixed; in Huang-Hilbert spectra on the other hand, this set is extracted from the signals themselves, which may lead to more accurate time-frequency representations.

In earlier work [3], we developed a synchrony measure for generic (one- and multi-dimensional) point processes ("event strings"), referred to as "stochastic event synchrony" (SES); we applied it to point processes on the time-frequency plane ("bump models") [4]. Those bump models capture the most prominent oscillatory events in the signals at hand (see Fig. 1). In [3], we used complex Morlet wavelets to map the signals unto the timefrequency plane. In this paper, the method of [3] is extended to Huang-Hilbert spectra [5]. One of the major advantages of the proposed approach is its simplicity: it avoids computing wavelets and extracting bump models, which are both computationally intensive operations.
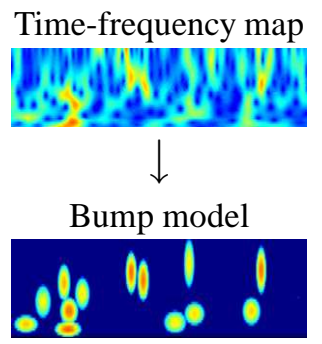

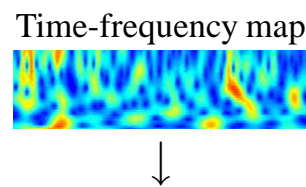

Bump model

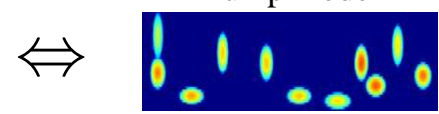

Fig. 1. SES applied to bump models [3].

The proposed method is applicable to generic time series; in this paper, however, we will focus on time series that occur in the context of neuroscience, in particular, electroencephalography (EEG) signals [6], recorded from multiple electrodes located on the scalp of a (human) subject. We will consider so called steady-state visually evoked potentials (SSVEP), which are stable responses that can be measured all over the scalp [7]. SSVEPs affect EEG synchrony, and as a result, synchrony measures can be applied to detect SSVEPs. In this paper, we will apply our novel synchrony measure to detect SSVEPs. We will present promising experimental results that seem to suggest that our method is substantially more sensitive to EEG synchrony perturbations than classical synchrony measures.

This paper is organized as follows. In the next section, we review the empirical mode decomposition (EMD) method and explain how it can be used to obtain time-frequency maps ("Huang-Hilbert spectra"). In Section 3, we outline the idea behind stochastic event synchrony, and discuss how it can be used to quantify the similarity of Huang-Hilbert spectra. In Section 4, we use this similarity measure to detect steady-state 
visually evoked potentials in EEG signals; we conclude this paper with comments on further potential applications of our approach.

\section{EMPIRICAL MODEL DECOMPOSITION (EMD)}

Empirical Mode Decomposition (EMD) decomposes signals into so called "intrinsic mode functions" (IMF) [5]. The latter are functions that satisfy the following two conditions: (i) the number of extrema and the number of zero crossings are either equal or differ at most by one; (ii) at any point, the mean value of the envelope defined by the local maxima and the envelope defined by the local minima is zero. An IMF represents an oscillatory mode within a given signal: its cycles (defined by its zero crossings) corresponds to one (and not more than one) mode of oscillation; both the amplitude and frequency of this oscillation may vary over time, in other words, the oscillation is not necessarily stationary nor narrow-band.

The process of extracting an IMF from a signal $x(t)$ ("sifting process" [5]) consists of the following steps: (i) determine the local maxima and minima of $x(t)$; (ii) generate the upper and lower signal envelope by connecting those local maxima and minima respectively by some interpolation method (e.g., linear, spline, piece-wise spline [5] [8]); (iii) determine the local mean $m(t)$, by averaging the upper and lower signal envelope; (iv) subtract the local mean from the data: $h_{1}(t)=$ $x(t)-m_{1}(t)$. Ideally, $h_{1}(t)$ is an IMF. However, in practice, $h_{1}(t)$ may still contain local asymmetric fluctuations, e.g., undershoots and overshoots; therefore, one needs to repeat the above four steps several times, resulting eventually in the first IMF. In order to obtain the second IMF, one applies the sifting process to the residue $\varepsilon_{1}(t)=x(t)-\mathrm{IMF}_{1}(t)$, obtained by subtracting the first IMF from $x(t)$; the third IMF is in turn extracted from the residue $\varepsilon_{2}(t)$ and so on. One stops extracting IMFs when two consecutive sifting results are close to identi$\mathrm{cal}$; the empirical mode decomposition of the signal $x(t)$ may be written as:

$$
x(t)=\sum_{k=1}^{n} \operatorname{IMF}_{k}(t)+\varepsilon_{n}(t),
$$

where $n$ is the number of extracted IMFs, and the final residue $\varepsilon_{n}(t)$ can either be the mean trend or a constant. An (artificial) example (from [9]) is illustrated in Fig. 2.

The empirical-mode decomposition is obviously complete, since (1) is an equality: the original signal can be reconstructed from the IMFs and the final residue. Note that the IMFs are not guaranteed to be mutually orthogonal, but in practice, they often are close to orthogonal; it is also noteworthy that the IMFs are adaptive, i.e., they depend on the signal $x(t)$, as we anticipated earlier.

By means of the IMFs, one can construct a time-frequency representation of the signal $x(t)$, i.e., the Huang-Hilbert spectrum (HHS) [5]. The idea is to compute the instantaneous amplitude and frequency of each IMF. This can be done by computing the following analytic signal from each IMF:

$$
Z(t)=\operatorname{IMF}(t)+i Y(t),
$$

with $Y(t)$ the Hilbert transform of the IMF:

$$
Y(t)=\frac{1}{\pi} \mathrm{P} \int_{-\infty}^{+\infty} \frac{\mathrm{IMF}(t)}{t-t^{\prime}} d t^{\prime}
$$

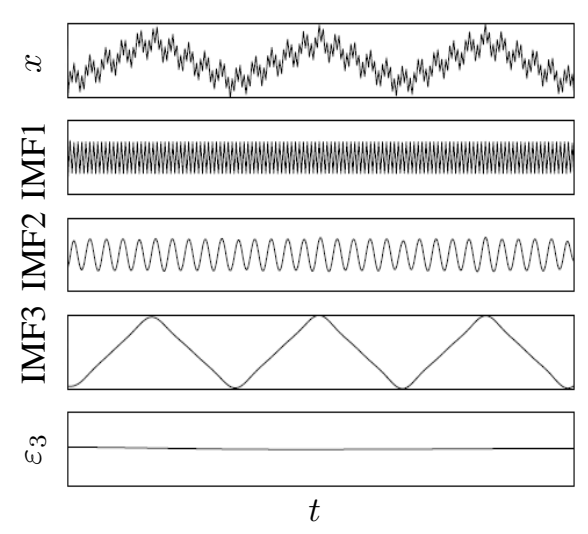

Fig. 2. Empirical mode decomposition (EMD): the original signal (top), three IMFs (middle), and residue (bottom) [9].

where $\mathrm{P}$ indicates the Cauchy principal value of the integral [10]. The instantaneous amplitude $a(t)$ and phase $\theta(t)$ of the IMF is defined as the magnitude and angle respectively of $Z(t)$. The instantaneous frequency is then simply defined as:

$$
\omega=\frac{d \theta(t)}{d t} \text {. }
$$

Huang-Hilbert spectra (HHS) represent the instantaneous amplitude against instantaneous frequency and time [5]. HHS images are typically sparse and contain sharp ridges, as illustrated in Fig. 3. In the following section, we propose a method to quantify the similarity of two Huang-Hilbert spectra; the key idea will be to match ridges in one HHS to ridges in the other HHS.
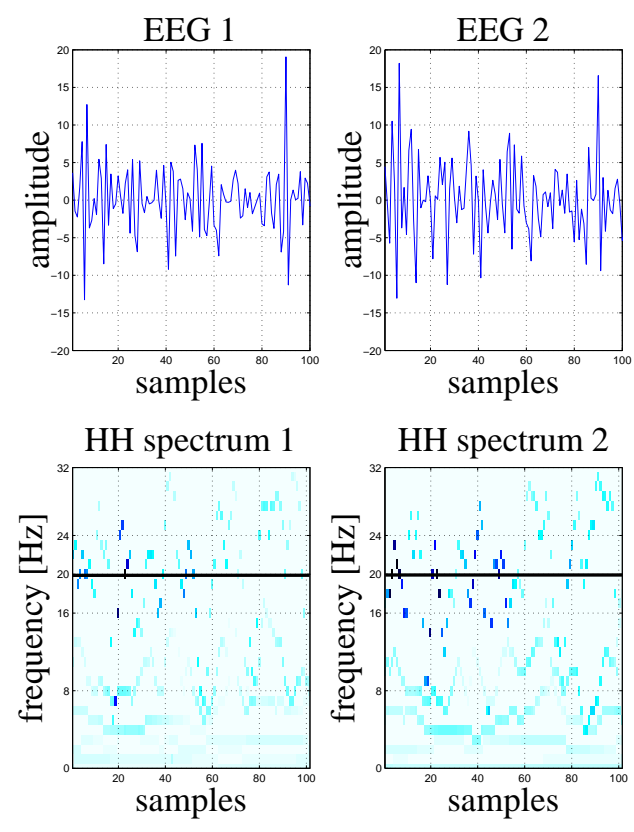

Fig. 3. Huang-Hilbert spectra (bottom) of two EEG channels (top); the horizontal line at $20 \mathrm{~Hz}$ in the $\mathrm{HH}$ spectra corresponds to the SSVEP stimulation frequency.

\section{STOCHASTIC EVENT SYNCHRONY (SES)}

In earlier work [3], we developed a measure that quantifies the similarity ("interdependence" or "synchrony") of two (one- 

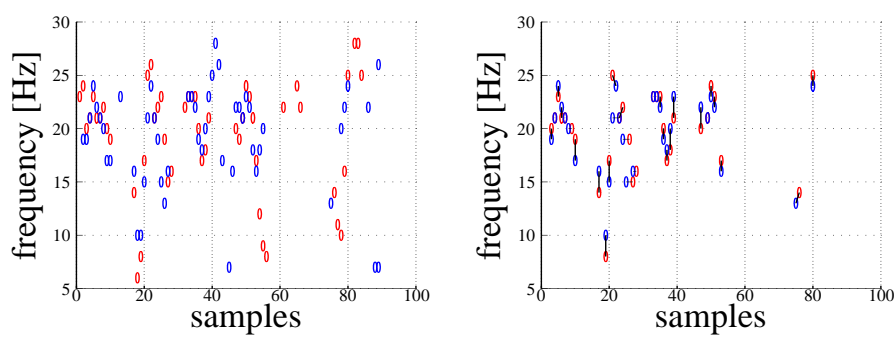

Fig. 4. (left) Events ("ridges") extracted from HHS1 (red) and HHS2 (blue) of Fig. 3; (right) coincident ridges.

or multi-dimensional) point processes, referred to as stochastic event synchrony (SES); we applied that measure to point processes on the time-frequency plane, more precisely, bump models [4] (cf. Fig. 1). Here we will use SES to quantify the similarity of two Huang-Hilbert spectra. The first step is to extract a point process ("event string") from an HHS (see Fig. 4 (left)): from the given HHS, we only retain the $N$ largest instantaneous amplitudes. ( $N$ is typically about 100 .) Each of the $N$ remaining points ("ridges") $r_{j}=\left(t_{j}, a_{j}, f_{j}\right)$ with $j=1, \ldots, N$ is viewed as an event, and the sequence $r=\left(r_{1}, \ldots, r_{N}\right)$ is a three-dimensional point process; in other words, the remaining points $r_{j}$ in Fig. 4 (left) take the role of the "bumps" in Fig. 1.

Fig. 4 (left) suggests a natural way to define the similarity of two HHS: ridges in one time-frequency map (red) may not be present in the other map (blue) ("non-coincident" ridges); other ridges are present in both maps ("coincident ridges"), but appear at slightly different positions on the maps.

Fig. 4 (right) depicts the coincident ridges, obtained after matching the event strings $r$ (red) and $r^{\prime}$ (blue); the black lines connect the centers of coincident ridges, and hence, they visualize the offset in position between pairs of coincident ridges. Stochastic event synchrony consists of the following parameters: (i) $\rho$ : fraction of non-coincident ridges; (ii) $\delta_{t}$ and $\delta_{f}$ : average time and frequency offset respectively between coincident ridges; (iii) $s_{t}$ and $s_{f}$ : variance of the time and frequency offset respectively between coincident ridges. The alignment of the two ridge traces (cf. Fig. 4 (right)) is cast as a statistical inference problem [3]. The associated probabilistic model depends on the SES parameters $\theta=\left(\delta_{t}, \delta_{f}, s_{t}, s_{f}\right)$ besides the following two kinds of latent variables: (i) binary variables $C_{k k^{\prime}}$, associated to each pair of ridges, where $C_{k k^{\prime}}=1$ indicates that event $S_{k}$ of the first HHS is coincident with event $S_{k^{\prime}}^{\prime}$ in the other HHS, and where $C_{k k^{\prime}}=0$ otherwise; (ii) binary variables $B_{k}$ and $B_{k^{\prime}}^{\prime}$, which indicate whether a ridge is non-coincident. The latent-variable model is of the form:

$$
\begin{aligned}
& p\left(r, r^{\prime}, b, b^{\prime}, c, \theta\right) \propto \\
& \prod_{k=1}^{n}\left(\beta \delta\left[b_{k}-1\right]+\delta\left[b_{k}\right]\right) \prod_{k^{\prime}=1}^{n^{\prime}}\left(\beta \delta\left[b_{k}^{\prime}-1\right]+\delta\left[b_{k}^{\prime}\right]\right) \\
& \cdot \prod_{k=1}^{n} \prod_{k^{\prime}=1}^{n^{\prime}}\left(\mathcal{N}\left(t_{k^{\prime}}^{\prime}-t_{k} ; \delta_{t}, s_{t}\right) \mathcal{N}\left(f_{k^{\prime}}^{\prime}-f_{k} ; \delta_{f}, s_{f}\right)\right)^{c_{k k^{\prime}}} \\
& \cdot \prod_{k=1}^{n}\left(\delta\left[b_{k}+\sum_{k^{\prime}=1}^{n^{\prime}} c_{k k^{\prime}}-1\right]\right) \prod_{k^{\prime}=1}^{n^{\prime}}\left(\delta\left[b_{k^{\prime}}^{\prime}+\sum_{k=1}^{n} c_{k k^{\prime}}-1\right]\right) \\
& \cdot p\left(\delta_{t}\right) p\left(s_{t}\right) p\left(\delta_{f}\right) p\left(s_{f}\right),
\end{aligned}
$$

where $\beta$ is a constant (which serves as a knob to control the number of non-coincident ridges), $n$ and $n^{\prime}$ is the total number of ridges in the two HHS, and $\mathcal{N}(x ; m, s)$ stands for a univariate Gaussian distribution with mean $m$ and variance $s$ [3]. For convenience, we choose improper priors $p\left(\delta_{t}\right)=p\left(\delta_{f}\right)=p\left(s_{t}\right)=$ $p\left(s_{f}\right)=1$. The SES parameters $\theta=\left(\delta_{t}, \delta_{f}, s_{t}, s_{f}\right)$ and the latent variables $C, B$ and $B^{\prime}$ are determined jointly by MAP estimation. This may practically be carried out by cyclic maximization [3]: for fixed $\theta$, one maximizes $\log p$ (cf. (5)) w.r.t. $C, B$ and $B^{\prime}$ and vice versa. Conditional maximization w.r.t. $\theta$ is straightforward, however, the conditional maximization w.r.t. $C, B$ and $B^{\prime}$ is non-trivial: it involves an intractable discrete optimization problem. We solve that problem approximately (but successfully) by iterative max-product message passing ("iterative dynamic programming") on a graphical model corresponding to the latent-variable probabilistic model (5) (see Fig. 5) [3]. The edges correspond to variables, the nodes corresponds to factors in (5). The nodes $\mathcal{N}$ corresponds to the Gaussian distributions in (5), the nodes denoted by $\bar{\Sigma}$ represent the factors $\left(\delta\left[b_{k}+\sum_{k^{\prime}=1}^{n^{\prime}} c_{k k^{\prime}}-1\right]\right)$ (blue) and $\left(\delta\left[b_{k^{\prime}}^{\prime}+\sum_{k=1}^{n} c_{k k^{\prime}}-1\right]\right)$ (red), and the nodes denoted by $\beta$ correspond to the factors $\left(\beta \delta\left[b_{k}-1\right]+\delta\left[b_{k}\right]\right)$ and $\left(\beta \delta\left[b_{k}^{\prime}-1\right]+\delta\left[b_{k}^{\prime}\right]\right)$. The arrows in Fig. 5 depict "messages" (i.e., probabilistic information about which pairs of ridges are coincident and which not) that are iteratively computed at each node according to the max-product computation rules. Intuitively, the nodes may be viewed as computing elements that iteratively update their opinion about which ridges match and which do not, based on the opinions ("messages") they receive from neighboring nodes. When the algorithm eventually has converged (and the nodes have found a consensus so to speak), the messages are combined to obtain a decision on $C, B$ and $B^{\prime}$, and an estimate of $\rho$ and the other SES parameters [3].

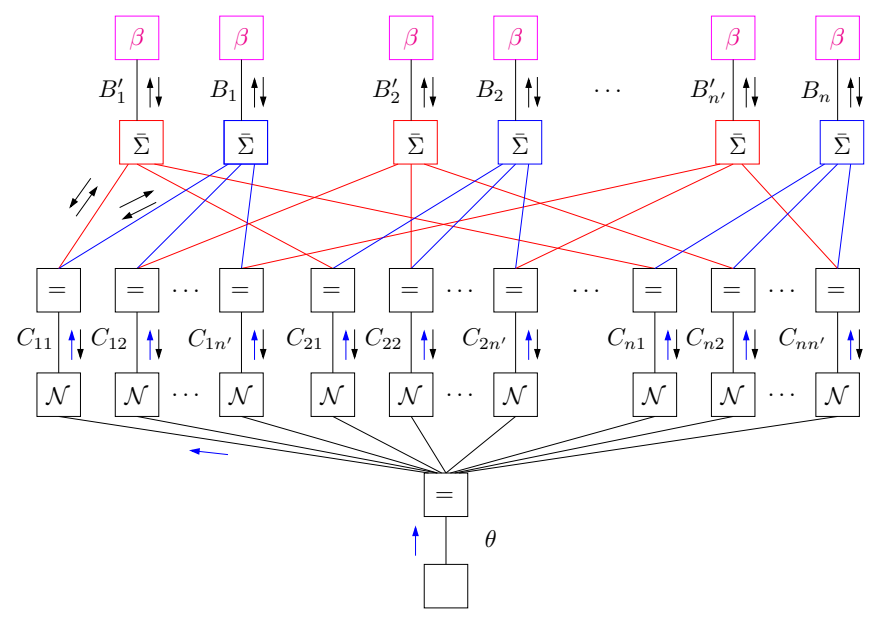

Fig. 5. Graphical model of (5).

\section{RESULTS}

We applied the proposed ridge-matching method to EEG signals; the latter were recorded from a human subject that was watching flashing patterns with frequency of $20 \mathrm{~Hz}$, presented during $2 \mathrm{~s}$ with silent intervals of $3 \mathrm{~s}$. The flashing patterns evoke oscillatory brain activity at the same frequency ("steady-state visually evoked potentials") [7]. The EEG signals were recorded from four locations (two frontal and two temporal areas) [6]. We used EMD to extract IMF components from those EEG signals (cf. Section 2). Next, the IMF components are Hilbert 
transformed (resulting in Huang-Hilbert spectra), and the lowfrequency drifts $(<5 \mathrm{~Hz})$ and high-frequency interference parts $(>30 \mathrm{~Hz})$ of the IMF components are removed. Figure 3 shows a segment of two EEG signals (recorded by two frontal channels) together with their Huang-Hilbert spectra. At last, we determine the similarity of the latter by means of stochastic event synchrony (cf. Section 3): each pair of HHS is matched, and the timing and frequency jitter $s_{t}$ and $s_{f}$ of the coincident ridges, and the fraction of non-coincident ridges $\rho$ are computed. Those parameters are then averaged over all pairs of Huang-Hilbert spectra, resulting in three global measures of interdependence. As a benchmark, we also quantify the synchrony of the EEG signals by means of classical measures: magnitude squared coherence $(\mathrm{COH})$, partial directed coherence (PDC), directed transfer function (DTF), direct DTF, and full frequency DTF [11]; those classical measures are computed after bandpass filtering the EEG signals in a narrow band $(18-22 \mathrm{~Hz})$ centered at the SSVEP stimulation frequency $(20 \mathrm{~Hz})$.

We investigated whether those measures can detect fluctuations in EEG synchrony ("interdependence") in the above experiment. In particular, we tried to distinguish EEG signals recorded during stimulation from EEG recorded in silent intervals; for each of those two conditions, we consider 32 EEG segments of the same length (1.5s). Table 1 summarized the results: it lists classification errors obtained by the leave-one-out procedure; for the sake of simplicity, we used a linear classifier (hyperplane). We used the synchrony measures as single features and as pairs of features. Due to space constraints, Table 1 only contains the results for the pairs that resulted in the smallest classification errors: of the pairs without SES parameters, the combination of dDTF and $\mathrm{COH}$ gave the best result $(21.8 \%$ incorrectly classified), whereas the best overall result was obtained by the combination of $\mathrm{COH}$ and $\rho(7.8 \%)$. This result could not be further improved by increasing the number of features; this is probably due to the relatively small size of the data set. On the other hand, from Fig. 6, it becomes clear that by using non-linear classification techniques such as support vector machines, one may be able to reduce the classification error even more.

\begin{tabular}{|l|c||l|c|}
\hline Features & Correct & Features & Correct \\
\hline \hline$s_{t}$ & $36.0 \%$ & $s_{f}$ & $29.7 \%$ \\
\hline$\rho$ & $\mathbf{1 4 \%}$ & $\mathrm{COH}$ & $25 \%$ \\
\hline PDC & $42.3 \%$ & DTF & $39.1 \%$ \\
\hline dDTF & $37.5 \%$ & ffDTF & $39.7 \%$ \\
\hline dDTF and $\mathrm{COH}$ & $21.8 \%$ & $\rho$ and $\mathrm{COH}$ & $\mathbf{7 . 8 \%}$ \\
\hline
\end{tabular}

Table 1. Percentage of incorrectly classified EEG signals.

\section{CONCLUSIONS}

We observed an increase in EEG synchrony during SSVEP stimulation, with the most significant effects measured by magnitude squared coherence $(\mathrm{COH})$ and the SES parameter $\rho$. Previous studies reported similar effects, and emphasized that the level of EEG synchrony is correlated with the subject's attention [12] [13].

The present study is highly relevant for brain-computer interfaces (BCI). A brain-computer interface is a direct communication pathway between a human or animal brain and an external device. Many studies have confirmed that SSVEP-based

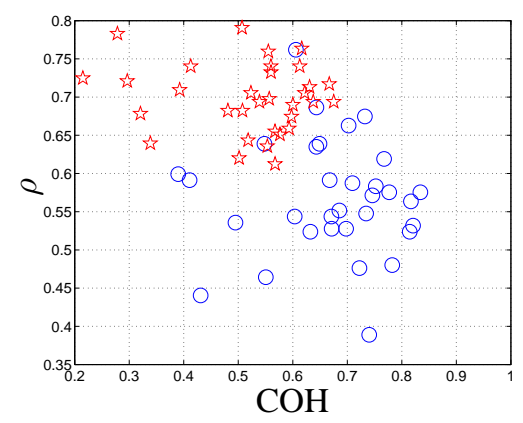

Fig. 6. $\rho$ vs. COH for SSVEP (blue circles) and non-SSVEP (red stars).

BCI systems have the highest overall performance; moreover, such systems can be used by most subjects without much prior training. However, state-of-the-art SSVEP-based BCI systems require long stimulation time to achieve satisfactory classification (usually around $3.5 \mathrm{~s}$ ), and therefore, they yield relatively low data rates. This is largely due to the fact that those systems typically only use the power spectrum as input features, in particular, the power spectrum at the SSVEP stimulation frequencies. As our study shows (see also [14]), EEG synchrony has strong potential for SSVEP detection: despite the very short stimulation period (only 1.5s), we obtained low classification errors $(7.8 \%$ at best). As a consequence, synchrony measures (particularly SES in conjunction with EMD) may in the future prove to be very useful in the context of BCI.

\section{REFERENCES}

[1] C. J. Stam, "Nonlinear Dynamical Analysis of EEG and MEG: Review of an Emerging Field," Clinical Neurophysiology 116:2266-2301 (2005).

[2] R. Q. Quiroga, A. Kraskov, T. Kreuz, and P. Grassberger, "Performance of Different Synchronization Measures in Real Data: A Case Study on EEG Signals," Physical Review E, vol. 65, 2002.

[3] J. Dauwels, F. Vialatte, T. Rutkowski, and A. Cichocki, "Measuring synchrony by message passing," in Neural Information Processing Systems (NIPS), Vancouver, Canada, December 3-6, 2007, to appear.

[4] F. Vialatte, C. Martin, R. Dubois, J. Haddad, B. Quenet, R. Gervais, and G. Dreyfus, "A Machine Learning Approach to the Analysis of Time-Frequency Maps, and Its Application to Neural Dynamics," Neural Networks, 2007, 20:194-209.

[5] N.E. Huang, Z. Shen, S.R. Long, M.C. Wu, H.H. Shih, Q. Zheng, N.-C. Yen, C.C. Tung, and H.H. Liu, "The empirical mode decomposition and the Hilbert spectrum for nonlinear and non-stationary time series analysis," Proceedings of the Royal Society A: Mathematical, Physical and Engineering Sciences, vol. 454, no. 1971, pp. 903-995, March 1998.

[6] E. Niedermeyer and F. L. Da Silva, Eds., Electroencephalography: Basic Principles, Clinical Applications, and Related Fields, Lippincott Williams \& Wilkins, 5th edition, 2004.

[7] K. Nakayama and M. Mackeben, "Steady state visual evoked potentials in the alert primate," Vision Res, vol. 22, no. 10, pp. 1261-1271, 1982.

[8] T. M. Rutkowski, A. Cichocki, and D. P. Mandic, Signal Processing Techniques for Knowledge Extraction and Information Fusion, chapter Information Fusion for Perceptual Feedback: A Brain Activity Sonification Approach, to appear. Springer, 2008.

[9] G. Rilling, P. Flandrin, P. Goncalves, "On Empirical Mode Decomposition and its Algorithms," Proc. IEEE-EURASIP Workshop on Nonlinear Signal and Image Processing, Grado, Italy, June 2003.

[10] Stephan L. Hahn, Hilbert Transforms in Signal Processing, Artech House, Boston - London, 1996.

[11] M. Kamiński and Hualou Liang, "Causal Influence: Advances in Neurosignal Analysis," Critical Review in Biomedical Engineering, 33(4):347-430 (2005).

[12] J. Ding, G. Sperling, and R. Srinivasan, "Attentional modulation of ssvep power depends on the network tagged by the flicker frequency," Cerebral Cortex, vol. 16, no. 7, pp. 1016-1029, 2006.

[13] R. Srinivasan, S. Petrovic, "MEG Phase Follows Conscious Perception during Binocular Rivalry Induced by Visual Stream Segregation." Cereb Cortex, 16: 597608, May 2006.

[14] E. Gysels, Phase synchronization for classification of spontaneous EEG signals in brain-computer interfaces, No. 3397, EPFL, Switzerland, 2005. 